\title{
Modeling time delays in the X-ray spectrum of the Seyfert galaxy MCG-6-30-15
}

\author{
R. W. Goosmann ${ }^{1,2}$, B. Czerny ${ }^{3}$, V. Karas ${ }^{1}$, and G. Ponti ${ }^{4,5}$ \\ 1 Astronomical Institute of the Academy of Sciences, Boční II 1401, 14131 Prague, Czech Republic \\ 2 Observatoire de Paris, Section de Meudon, LUTH, 5 place Jules Janssen, 92195 Meudon Cedex, France \\ Copernicus Astronomical Center, Bartycka 18, 00-716 Warsaw, Poland \\ Dipartimento di Astronomia, Università di Bologna, via Ranzani 1, 40127 Bologna, Italy \\ 5 INAF-IASF Bologna, via Gobetti 101, 40129, Bologna, Italy
}

Received 20 July 2006 / Accepted 12 February 2007

ABSTRACT

\begin{abstract}
Aims. We propose a reflection model of the time delays detected during an exceptionally bright, single flare in MCG-6-30-15. We consider a scenario in which the delays of the hard X-rays with respect to the soft X-rays are caused by the presence of the delayed reflection component.

Methods. We employ a model of the flare, which is accompanied by reprocessed emission. We consider two geometries/thermal states of the reprocessing medium: a partially ionized accretion disk surface and a distribution of magnetically confined, cold blobs.

Results. The reprocessing by cold blobs predicts positive time delays and a saturation in the time delay - energy relation, which is likely present in the data. The model requires a strong reflection component and relies on the apparent pivoting of the combined primary and reflected spectrum. The reflection by the ionized disk surface does not reproduce the observed delays. We discuss the relation between the two reflection scenarios and argue that they are both present in MCG-6-30-15.
\end{abstract}

Key words. accretion, accretion disks - galaxies: Seyfert - galaxies: active - radiative transfer - X-rays: galaxies

\section{Introduction}

It has been known for a long time that Active Galactic Nuclei (AGN) show strong non-periodic variability in the X-ray band on timescales ranging from years down to hours and minutes (e.g. Lawrence et al. 1987; McHardy \& Czerny 1987; Mushotzky et al. 1993; Vaughan et al. 2003a; Kaastra et al. 2004).

The predominant power-law character of the X-ray emission of most Seyfert galaxies and quasars can be successfully modeled assuming Compton scattering of soft photons by a hot plasma (e.g. Shapiro et al. 1976; Sunyaev \& Titarchuk 1980). In the accretion disk scenario of AGN the soft photons are naturally provided by the disk emission whilst the plasma can be identified with a hot corona of a temperature $\sim 10^{9} \mathrm{~K}$. The detection of the high energy cut-off in a number of radio quiet AGN, with values between 60 and $300 \mathrm{keV}$ (e.g. Zdziarski et al. 1995; Risaliti 2002; Deluit \& Courvoisier 2003; Soldi et al. 2005; Beckmann et al. 2005), supports the thermal origin of the X-ray emission in these sources.

The power-law shape of the X-ray spectra is a consequence of successive Compton up-scatterings. This should lead to systematic time delays between the hard and the soft X-ray variations as the hard $\mathrm{X}$-ray photons must undergo more scattering events. A search for such delays was performed for MCG-6-3015 and other Seyfert galaxies but the results are not unique.

Reliable measurements of X-ray time delays in AGN spectra require sufficiently long observation times. For a $95 \mathrm{ks}$ observation of MCG-6-30-15 with XMM-Newton the quality of the data did not allow to confirm any spectral delays (Ponti et al. 2004). They were only reported after a follow-up 300 ks observation when Vaughan et al. (2003b) detected delays by $200 \mathrm{~s}$ on timescales of $10^{4} \mathrm{~s}$. Frequency-dependent time delays were found in a number of sources (NGC 7469, Papadakis et al. 2001; NGC 4051, McHardy et al. 2004; MCG-6-30-15, Vaughan et al. 2003b; NGC 3783, Markowitz 2005; Ark 564, Arévalo et al. 2006 ), for a frequency range of $10^{-6}$ to $10^{-3} \mathrm{~Hz}$. These delays turn out to be roughly proportional to the Fourier period and they increase with the energy span considered (max. between 0.2 to $12 \mathrm{keV}$ ) up to a few thousand seconds.

The dependence of the delay time on the Fourier frequency cannot be accommodated within the frame of a simple Comptonization model that assumes electron clouds of a uniform density and temperature. In such models the observed hard-to-soft time delay is independent of the Fourier frequency (Miyamoto et al. 1988). Therefore, two types of more sophisticated models were proposed:

- an extended Comptonizing region with the appropriate density and temperature stratification (extended spherical region, Kazanas et al. 1997; advection-dominated inflowoutflow model, Blandford \& Begelman 1999; extended coronal emission model, Arevalo \& Uttley 2006)

- models of a local spectral evolution due to coherent changes in a single compact emission region (single coronal flare evolution, Poutanen \& Fabian 1999; Życki 2003; inflow of a single blob, Böttcher \& Liang 1999; Życki 2004)

These models predict a pivoting of the hard X-ray spectrum, and the pivoting around the appropriate frequency can explain the apparent time delays, as argued by Körding \& Falcke (2004).

The detection of a single gigantic flare in the lightcurve of MCG-6-30-15 (Ponti et al. 2004) offers a specific insight into 
the delay problem assuming that in this exceptional case we see a localized flare event (instead of a random peak in the net flux of large flare distributions). This assumption is inspired by the rather symmetric shape of the lightcurve during the flare event and by its short time scale of only 2200 s. Ponti et al. (2004) detected spectral time delays during the flare period and found that the hard X-rays were lagging the soft band. They interpret this as a result of multiple photon scattering within the flare itself.

Using the same data, we discuss in this paper an alternative possibility to explain the time delays. While the models listed above focus primarily on the Compton up-scattering in the hot plasma, we investigate a possible role of the reprocessed component for the observed time delays. Unlike the primary, the reprocessed radiation arises in a relatively cooler medium and thus has a different spectral shape. The spatial distance between the primary source and the reprocessing site causes a delay between the two components and a time evolution of the combined (i.e. primary plus reflection) spectrum. We will show that this leads to an apparent effect of energy-dependent time delays. We investigate two types of reprocessing: a strongly ionized reflector and a weakly ionized, cold reflector.

After recalling the analysis of the data (Sect. 2) we describe a simple model (Sect. 3) to find general trends of the spectral time delays between various energy bands. We discuss their dependence on details of the model setup for the two reprocessing scenarios (Sect. 4). We then show how the spectral delays of the observed flare can be reproduced by our model (Sect. 5) and we discuss and summarize our results (Sect. 6).

\section{Observation and data reduction}

The data were collected during the observation performed on June 11th, 2000, with the EPIC MOS1, MOS2 and pn cameras of XMM-Newton (Strüder et al. 2001) operated in timing, fullframe, and small window modes. The source was observed for $95 \mathrm{ks}$ and the spectral data were first published in Wilms et al. (2001) whilst Ponti et al. (2004) performed a timing analysis.

The data were re-reduced and screened using the SAS software v6.5.0. We only used the pn data as there was no significant pile-up present. The single and double pixel events (i.e. pattern $\leq 4)$ were selected. Source and background counts were extracted from two circular regions of $45^{\prime \prime}$ radius. The background events were taken from a region far from the source but within the same chip.

During the observation MCG-6-30-15 showed a bright flare lasting for $\sim 2000 \mathrm{~s}$. The lightcurve over the flare period is shown in Fig. 1 (top). It was decomposed into six lightcurves in separate energy bands, and X-ray time delays between them were calculated from cross-correlation functions (Ponti et al. 2004). The delays increase with difference in energy (Fig. 1, bottom). The photons at $10 \mathrm{keV}$ lag by a few hundred seconds with respect to those below $0.57 \mathrm{keV}$.

\section{The model}

We assume that the flare is created in a compact region at some distance from the reprocessing matter. The resulting primary component $I_{\mathrm{p}}$ partly shines directly toward a distant observer and partly gives rise to the Compton reflection/reprocessed component $I_{\mathrm{r}}$. The response of $I_{\mathrm{r}}$ to spectral variations of $I_{\mathrm{p}}$ is delayed by the light travel time between the flare source, the reprocessing sites, and the observer. This time delay depends on the geometry in the vicinity of the flare and also on the viewing angle.
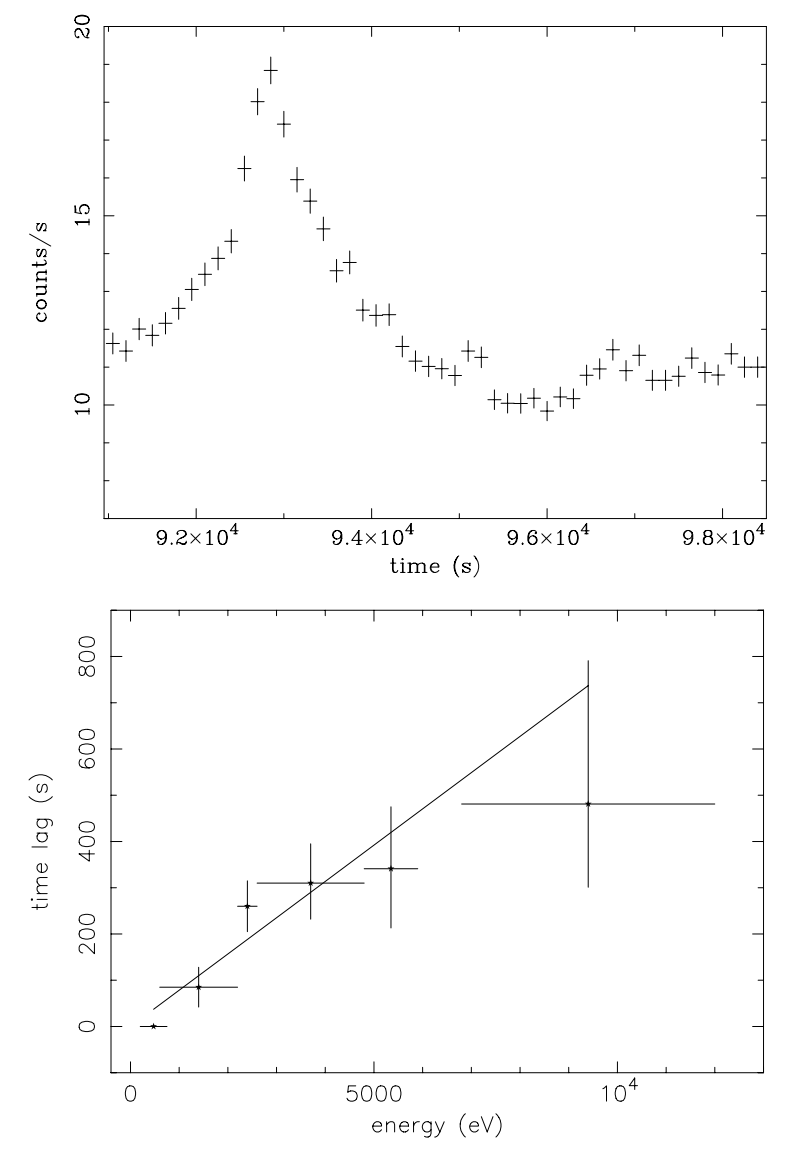

Fig. 1. Top: the light-curve over $0.2-10 \mathrm{keV}$ during the period of the strong flare in MCG-6-30-15. Bottom: energy-dependent time delays computed by Ponti et al. (2004) together with a linear fit representing Comptonization inside the flare.

An extended reprocessing region should lead to some level of smearing of the original signal, i.e. the duration of the brightening of the reflected component will be longer than the duration of the flare itself.

We introduce a simple mathematical parameterization of $I_{\mathrm{p}}$ and $I_{\mathrm{r}}$ as a function of energy $E$ and time $t$. For both components we assume a power-law dependence in energy with indexes $\alpha_{\mathrm{p}}$ and $\alpha_{\mathrm{r}}$. For $I_{\mathrm{r}}$ a broken power-law is also possible. It involves two branches with indexes $\alpha_{\mathrm{r}_{1}}$ and $\alpha_{\mathrm{r}_{2}}$ that connect at the break energy $E_{\mathrm{br}}$. This parameterization neglects particular reprocessing features, such as the emission lines, but represents rather well the overall shape of the continuum for a neutral or partially ionized reflector.

We imagine the time modulation of $I_{\mathrm{p}}$ and $I_{\mathrm{r}}$ as consecutive events. They are modeled by Lorentzians, $\mathcal{L}_{\mathrm{p}}(t)$ and $\mathcal{L}_{\mathrm{r}}(t)$, which take the half-width parameter $T_{\mathrm{f}}$ and the time $t_{0}$ marking the maximum of the primary emission. The primary leads the reprocessed spectrum by a delay $\delta$ giving a measure of the average light travel time between the flare source and the reprocessing sites. As there are many possible light paths for the reprocessing the width of $\mathcal{L}_{\mathrm{r}}$ can be broadened by a factor $b$. Note that this broadening is also influenced by the viewing angle of the distant observer. The equations for $I_{\mathrm{p}}$ and $I_{\mathrm{r}}$ read as follows:

$$
\begin{aligned}
& I_{\mathrm{p}}(E, t)=\mathcal{L}_{\mathrm{p}}(t) E^{-\alpha_{\mathrm{p}}} \\
& I_{\mathrm{r}}(E, t)=N \mathcal{L}_{\mathrm{r}}(t) \times \begin{cases}E^{-\alpha_{\mathrm{r}_{1}}} & \text { for } E \leq E_{\mathrm{br}}, \\
E_{\mathrm{br}}^{\alpha_{\mathrm{r}_{2}}-\alpha_{\mathrm{r}_{1}}} E^{-\alpha_{\mathrm{r}_{2}}} & \text { for } E>E_{\mathrm{br}},\end{cases}
\end{aligned}
$$


where

$\mathcal{L}_{\mathrm{p}}(t)=\frac{T_{\mathrm{f}}^{2}}{\left(t-t_{0}\right)^{2}+T_{\mathrm{f}}^{2}}$,
$\mathcal{L}_{\mathrm{r}}(t)=\frac{b^{2} T_{\mathrm{f}}^{2}}{\left(t-t_{0}-\delta\right)^{2}+b^{2} T_{\mathrm{f}}^{2}}$.

A factor $N$ is included in $I_{\mathrm{r}}$ to normalize the reflected component against the primary. We define $N$ with respect to the ratio, $K$, between $I_{\mathrm{p}}$ and $I_{\mathrm{r}}$ integrated over time and energy:

$K=\frac{N b\left[\int_{E_{\min }}^{E_{\mathrm{br}}} \mathrm{d} E^{\prime} E^{\prime-\alpha_{\mathrm{r}_{1}}}+\int_{E_{\mathrm{br}}}^{E_{\max }} \mathrm{d} E^{\prime \prime} E^{\prime \prime-\alpha_{\mathrm{r}_{2}}}\right]}{\int_{E_{\min }}^{E_{\max }} \mathrm{d} E E^{-\alpha_{\mathrm{p}}}}$.

with $b=\left[\int \mathcal{L}_{\mathrm{r}}\left(t^{\prime}\right) \mathrm{d} t^{\prime}\right] /\left[\int \mathcal{L}_{\mathrm{p}}(t) \mathrm{d} t\right]$. For a given value of $K$, the normalization $N$ is a function of the energy limits, the broadening, and the spectral indexes. A distant observer detects the sum

$I_{\mathrm{obs}}(E, t)=I_{\mathrm{p}}(E, t)+I_{\mathrm{r}}(E, t)$.

We specify the energy bands, $\Delta E_{i}$, with $i=1, \ldots, 6$, and construct light-curves

$L_{i}(t)=\int_{\Delta E_{i}} I_{\mathrm{obs}}(E, t) \mathrm{d} E$.

To investigate the time delay between the spectral response in different energy bands we compute cross-correlation functions

$F_{\mathrm{CCF}}^{i j}(\tau)=\frac{\int_{-\tau_{\max }}^{+\tau_{\max }} L_{i}(t) L_{j}(t-\tau) \mathrm{d} t}{\sqrt{\int_{-\tau_{\max }}^{+\tau_{\max }} L_{i}^{2}\left(t^{\prime}\right) \mathrm{d} t^{\prime}} \times \sqrt{\int_{-\tau_{\max }}^{+\tau_{\max }} L_{j}^{2}\left(t^{\prime \prime}\right) \mathrm{d} t^{\prime \prime}}}$,

where the denominator is a normalization factor and $\tau_{\max }$ must be large enough for $F_{\mathrm{CCF}}^{i j}(\tau)$ to converge. The global maximum of $F_{\mathrm{CCF}}^{i j}(\tau)$ marks the time delay between the energy bands $\Delta E_{i}$ and $\Delta E_{j}$.

\section{Results}

Our model contains various parameters. Some of them are fixed by the flare observation described in Ponti et al. (2004). The total energy range, and the energy channels are specified as in the analysis: $E_{\min }=0.2 \mathrm{keV}, E_{\max }=12 \mathrm{keV}$ and the energy bands are listed in Table 1. We adopt the time of the flare maximum at $t_{0}=2200 \mathrm{~s}$ and the half width $T_{\mathrm{f}}=500 \mathrm{~s}$. The slope of the primary emission is not known very accurately due to the complex X-ray spectrum of MCG-6-30-15. It correlates with the hard X-ray flux, and rises up to $\sim 1.3$ (Vaughan \& Edelson 2001; Shih et al. 2002). We thus choose $\alpha_{\mathrm{p}}=1.3$ as the upper limit of the slope. As the lower limit we set $\alpha_{\mathrm{p}}=0.9$, which is consistent with Beppo-SAX data suggesting slopes $\sim 0.94$ (Fabian et al. 2002a), and also with the average slope in a large sample of radio-quiet AGN (Nandra et al. 1997; George et al. 2000; Piconcelli et al. 2005). The fixed parameters of the model are summarized in Table 1.
Table 1. Energy bands and fixed parameters of the model.

\begin{tabular}{cc|cc}
\hline \hline Energy band & Range $[\mathrm{keV}]$ & Fixed parameter & Value \\
\hline$\Delta E_{1}$ & $0.2-0.57$ & $E_{\min }$ & $0.2 \mathrm{keV}$ \\
$\Delta E_{2}$ & $0.6-2.2$ & $E_{\max }$ & $12 \mathrm{keV}$ \\
$\Delta E_{3}$ & $2.2-2.6$ & $\alpha_{\mathrm{p}}$ & $0.9,1.3$ \\
$\Delta E_{4}$ & $2.6-4.8$ & $t_{0}$ & $2200 \mathrm{~s}$ \\
$\Delta E_{5}$ & $4.8-6.8$ & $T_{\mathrm{f}}$ & $500 \mathrm{~s}$ \\
$\Delta E_{6}$ & $6.8-12.0$ & & \\
\hline
\end{tabular}

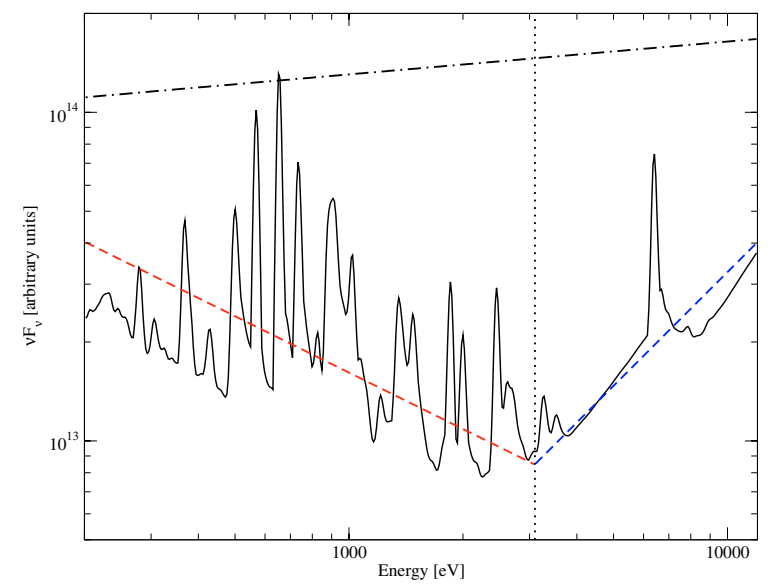

Fig. 2. An example of ionized reflection: the primary $F_{\mathrm{p}}(E) \propto E^{-0.9}$ (dot-dashed) and the local reprocessing (solid) of the ionized reflector scenario. The dashed line denotes the power-law fit $F_{\mathrm{r}}(E) \propto E^{-\alpha_{\mathrm{r}}}$ adopted for the delay modeling. Below $3.1 \mathrm{keV}$ the slope $\alpha_{\mathrm{r}_{1}}=1.57$ and above $\alpha_{\mathrm{r}_{2}}=-0.15$.

\subsection{Delays for a highly ionized reflector}

Highly ionized reflection is likely to form when a strong flare is localized not far from the accretion disk. An example of the reflected spectrum for such a case is shown in Fig. 2. The spectrum is calculated using the codes TITAN and NOAR (Dumont et al. 2000, 2003). The disk vertical structure is determined with an improved version of the code given in Różańska et al. (2002). We assume $M=10^{7} M_{\odot}$ (McHardy et al. 2005) and $a / M=0.998$ (Fabian et al. 2002a; Miniutti et al. 2003; Fabian \& Vaughan 2003). The flare is located at the disk radius $r=18 R_{\mathrm{g}}$.

The reflected component has a typical spectral shape for reprocessing by highly ionized material: it is relatively hard above $\sim 3 \mathrm{keV}$ and steep (soft) at lower energies:

$I_{\mathrm{r}}(E, t)=N \mathcal{L}_{\mathrm{r}}(t) \times \begin{cases}E^{-1.57} & \text { for } E \leq 3.1 \mathrm{keV} \\ E^{+0.15} & \text { for } E>3.1 \mathrm{keV}\end{cases}$

The relative normalization of the primary and the reflected component is taken from the modeling shown in Fig. 2. We obtain $K \sim 0.15$ if the flare radiates isotropically. With $b=2$ this leads to a normalization of $N \sim 0.022(N \sim 0.025)$ for $\alpha_{\mathrm{p}}=0.9$ $\left(\alpha_{\mathrm{p}}=1.3\right)$. However, large values of the equivalent width of the iron line (Wilms et al. 2001; Reynolds et al. 2004) indicate that the reflection may be strongly enhanced with respect to isotropic emission (e.g. Fabian et al. 2002a; Ballantyne et al. 2003; Taylor et al. 2003). Therefore, we keep $N$ as a free parameter of the model.

We vary $N$ between 0.01 and 0.9 and set the intrinsic delay $\delta$ to $500 \mathrm{~s}, 700 \mathrm{~s}, 1000 \mathrm{~s}$ and $1700 \mathrm{~s}$. The spectral time delays at all energy bands $\Delta E_{i}$ are obtained and normalized so that they are measured with respect to the one at $\Delta E_{1}$. The same normalization was applied in the data analysis (Ponti et al. 2004). 

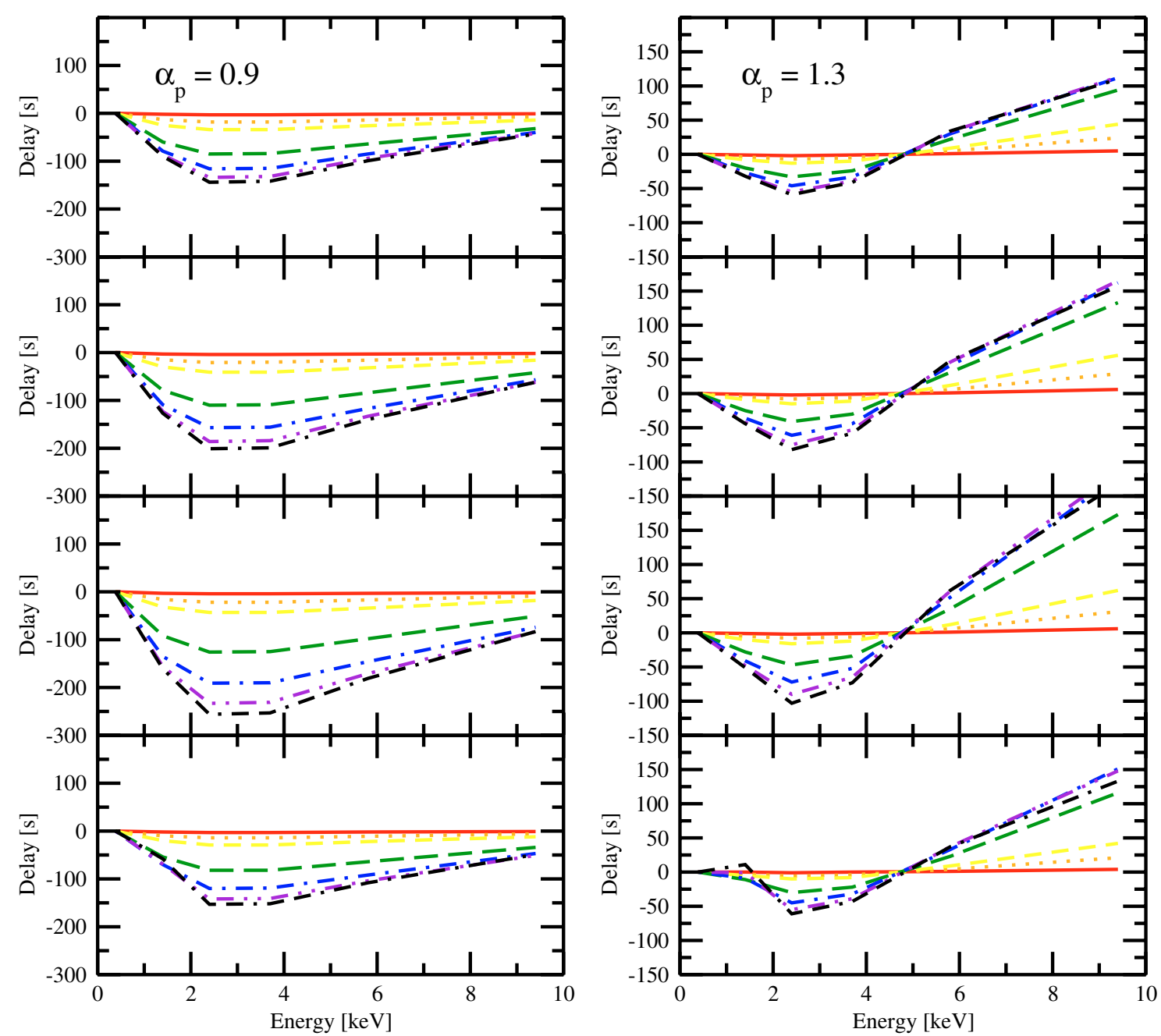

Fig. 3. Energy-dependent time delays for a highly ionized reflector with two slopes of the primary spectrum. Left: $\alpha_{\mathrm{p}}=0.9$, right: $\alpha_{\mathrm{p}}=1.3$. From top to bottom the panels represent intrinsic delays $\delta=500 \mathrm{~s}, 700 \mathrm{~s}, 1000 \mathrm{~s}$, and $1700 \mathrm{~s}$. The different curves denote the following values for $N$ : 0.01 (red, solid), 0.05 (orange, dots), 0.1 (yellow, short dashes), 0.3 (green, long dashes), 0.5 (blue, dashes/dots), 0.7 (pink, dashes/double-dots), and 0.9 (black, double-dashes/dots). Note that the vertical scale is different for both columns.

The results for a broadening of $b=2$ and for the two values of $\alpha_{\mathrm{p}}$ are shown in Fig. 3.

Obviously, for the very low normalization values of $N=$ 0.022 or $N=0.025$ no significant time delays appear. Noticeable delays only exist for $N$ being higher by at least one order of magnitude. But even at higher $N$ these delay curves are very different from the ones obtained by Ponti et al. (2004). The model mostly produces negative delays, while the observed ones are always positive.

The behavior of the delay curves as a function of $N$ and $\delta$ is partly analogous in both cases of $\alpha_{\mathrm{p}}$. The overall normalization of the curves increases with $\delta$ until a maximum at $\sim 1000 \mathrm{~s}$ is reached, then, for even higher $\delta$, the normalization decreases again. It also rises with $N$ but saturates when $N$ goes beyond unity.

There are also some important differences between the two cases of $\alpha_{\mathrm{p}}$. For $\alpha_{\mathrm{p}}=0.9$ the delays are negative at all energies and the shape of the curves is concave over the whole energy range. The minimum delay occurs around $3 \mathrm{keV}$. In the case of $\alpha_{\mathrm{p}}=1.3$ the shortest delays appear at a slightly lower value. The curves rise more quickly at intermediate energies and their curvature becomes convex around $5 \mathrm{keV}$. At high energies, positive delays are obtained.
We vary the broadening parameter $b$ while fixing the timeintegrated energy output of the reprocessed component. Thus, for each value of $b$ we find the corresponding $N$ from Eq. (4). We consider seven values for $b$ and $N$ with $N b=1.8$. The resulting delay curves for $\alpha_{\mathrm{p}}=0.9$ and $\delta=1000 \mathrm{~s}$ are shown in Fig. 4. For a given value of $\mathrm{Nb}$ a whole range of curves is obtained. However, the characteristic concave curvature and the minimum around $3 \mathrm{keV}$ are always preserved. Conducting the same investigation for other values of $N b$ delivers similar results. Therefore, varying $b$ does not help to overcome the discrepancy between the modeled time delays and those observed during the flare in MCG-6-30-15.

\subsection{Delays for a weakly ionized/neutral reflector}

In our second scenario we investigate the time delays obtained for a weakly ionized reflector. This is a likely situation when the flare is located far from the accretion disk and/or the cold plasma is magnetically confined and denser than the plasma at the surface of an irradiated standard disk.

As a specific example of the reprocessed component we use the model of irradiated magnetically confined, cold clumps. The radiative transfer calculations for such a medium were 


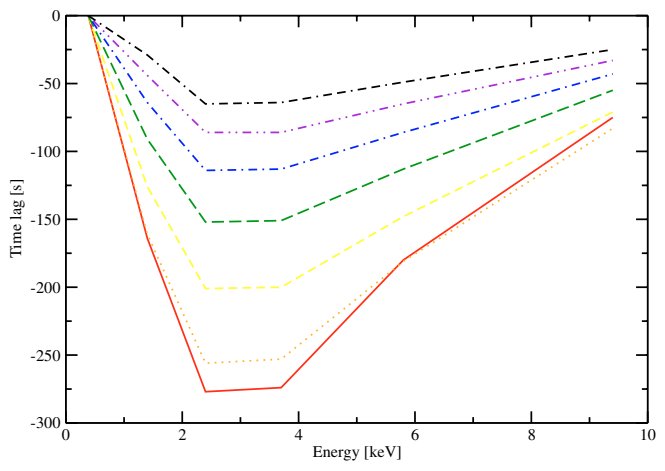

Fig. 4. Time delay curves derived for various values of $b$ in the scenario of a highly ionized reflector. The normalization $N b$ of the total energy released is kept constant at $N b=1.8$. The bottom curve (red, solid) represents the value $b=1.5$. From bottom to top, the value increases in steps of 0.5 for each curve and ends up at $b=4.5$ for the highest curve (black dots/double-dashes).

performed by Kuncic et al. (1997). An important effect in their modeling is that successive reprocessing can significantly lower the spectral energy in the soft X-ray range leading to very hard spectral slopes of $I_{\mathrm{r}}$. This hardening depends on the number density of the clouds in the medium. Here we adopt the parameterization

$I_{\mathrm{r}}=N \mathcal{L}_{\mathrm{r}}(t) E^{-0.1}$,

which is inferred from the case of 50 individual clouds on the line of sight. The index $\alpha_{\mathrm{r}}=0.1$ is taken from fitting the corresponding curve in Fig. 2 of Kuncic et al. (1997) between $0.2 \mathrm{keV}$ and $12 \mathrm{keV}$ with a power-law. We compute delay curves for $\delta$ set to $500 \mathrm{~s}, 700 \mathrm{~s}, 1000 \mathrm{~s}$, and $1700 \mathrm{~s}$. The values of the normalization $N$ are taken between 0.01 and 0.9 and the broadening parameter is set to $b=2$. The results are shown in Fig. 5 .

As for the highly ionized reflector the very low values of $N=0.022$ and $N=0.025$ lead to no significant time delays. But for higher $N$ the curves look very different from the previous scenario. They resemble a lot more the observed delays found by Ponti et al. (2004). Note that strong reflection was also required to model the energy-dependent fractional variability amplitude in MCG-6-30-15 (Goosmann et al. 2006), and it is consistent with the large value of the iron line equivalent width measured by several authors (e.g. Wilms et al. 2001; Reynolds et al. 2004). Therefore, the high value of $N$ may be justified.

The following trends can be derived from the model: the overall normalization of the time delay curves increases with $\delta$, goes through a maximum around $\delta=1000 \mathrm{~s}$ and then decreases again. All curves have positive slopes and the spectra evolve from softer to harder X-ray energies. The shape of the curves changes with $N$. Higher values of $N$ lead to longer delays until $N$ passes unity and the curves saturate at a level, which scales with $\delta$. For the steeper primary spectrum with $\alpha_{\mathrm{p}}=1.3$ the delay curves show the same general features and tendencies as for $\alpha_{\mathrm{p}}=0.9$. However, for $\alpha_{\mathrm{p}}=1.3$ the overall normalization of the time delay curves is larger and the curves are generally steeper in the soft X-ray range.

We investigate the influence of $b$ in a series of calculations with $\alpha_{\mathrm{p}}=0.9, N b=1.8$, and $\delta=1000 \mathrm{~s}$. The resulting time delay curves are shown in Fig. 6. For increasing values of $b$, the delays become smaller. This can partly be explained by the lower normalization $N$ required to keep the same value of $N b=1.8$. It was shown above that the delay curves level down for lower values of $N$. Another point is that for larger $b$ the variations of $I_{\mathrm{r}}$ start earlier in time. Thus, the variations of $I_{\mathrm{p}}$ are less ahead of the variations of $I_{\mathrm{r}}$. As for the highly ionized reflector, conducting the same investigation for other values of $\mathrm{Nb}$ delivers similar results.

\section{Modeling the measured time delays of MCG-6-30-15}

As shown in Fig. 3, the highly ionized reflector only predicts time delays that are non-monotonic with energy. This is due to the concave reflection spectrum of the scenario. On the other hand, a cold reflector with a hard single power-law spectral shape gives a monotonic increase. Certain parameter sets of the latter scenario reproduce the observed time delays for the flare of MCG-6-30-15 quite well. In Fig. 7 we show two examples of satisfactory data representation. The model reproduces the curvature seemingly present in the data. This may indicate that it catches the apparent signal propagation better than the fit by a straight line used in Ponti et al. (2004) and Fig. 1 (bottom). The primary slope of the two solutions shown was set to $\alpha_{\mathrm{p}}=1.3$ assuming that for the high flux state of the flare the spectrum should steepen (Vaughan \& Edelson 2001; Shih et al. 2002).

The solution is not unique. Partly, this is due to the large error bars attached to the data points in Fig. 7. There are three free parameters involved: the normalization $N$, the broadening $b$, and the intrinsic delay $\delta$. For higher accuracy of this modeling, the absolute normalization $K$ of the reprocessed power with respect to the primary must be determined in an independent way. Then the value of $N b$ can be computed from Eq. (4) (which links $N$ and $b$ ). Obtaining $K$ for the specific case of the observed flare in MCG-6-30-15 would require a very detailed spectral analysis. A decomposition into primary radiation and multiple reprocessing components using a sophisticated radiative transfer model would be helpful. However, the flare period only lasted for $\sim 2000 \mathrm{~s}$ and the obtained spectra are not of a sufficient accuracy to allow such an investigation.

Nevertheless, we are able to give a few robust conclusions. The model indicates that an intrinsic time delay of $1000 \mathrm{~s}$ is required. Lower or higher values of $\delta$ do not deliver any satisfying fits. The delay of $1000 \mathrm{~s}$ translates to a light travel distance of $\sim 60 R_{\mathrm{g}}$, assuming a black hole mass $M=3.3 \times 10^{6} M_{\odot}$ (McHardy et al. 2005). The broadening factor $b=2.5$ suggests a significant spread in the distances between the source and the reprocessor which would agree with the multiple scattering expected in the clumpy scenario. The lower limit of the normalization is at $N=0.7$, hence the reprocessing has to be strong. In the cold reflection model this implies a high covering factor of the flare source.

\section{Discussion and summary}

We considered two scenarios in which the time delays of the hard to the soft X-rays are caused by the delayed reflection component. The dependence of the delay on energy is caused by a difference in the spectral shape between the primary and the reprocessed spectrum. In the first scenario the reflector is ionized, the reflection component is concave, the predicted time delays are non-monotonic with energy, and the observed time delays are not reproduced. In the second scenario the reflection spectrum is close to a single hard power-law and the predicted time delays increase with energy. In this case, the model reproduces qualitatively the observed time delay curve. 

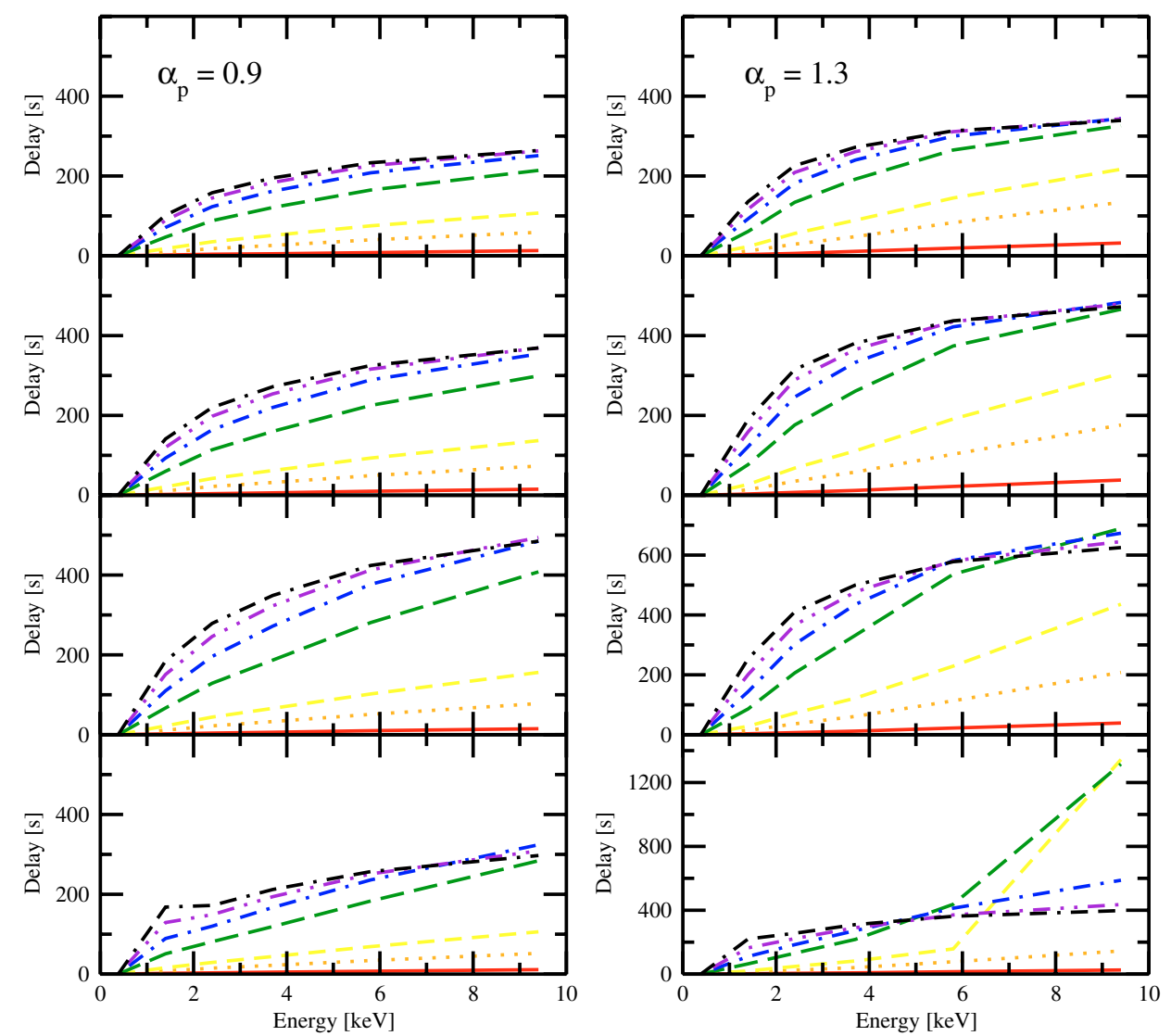

Fig. 5. Energy-dependent time delays for a weakly ionized/neutral reflector for two slopes of the primary spectrum. Left: $\alpha_{\mathrm{p}}=0.9$, right: $\alpha_{\mathrm{p}}=1.3$. From top to bottom the panels represent intrinsic delays $\delta=500 \mathrm{~s}, 700 \mathrm{~s}, 1000 \mathrm{~s}$, and $1700 \mathrm{~s}$. The different curves denote the following values for $N$ : 0.01 (red, solid), 0.05 (orange, dots), 0.1 (yellow, short dashes), 0.3 (green, long dashes), 0.5 (blue, dashes/dots), 0.7 (pink, dashes/double-dots), and 0.9 (black, double-dashes/dots). Note the change in vertical scale for the last two panels of the right column.

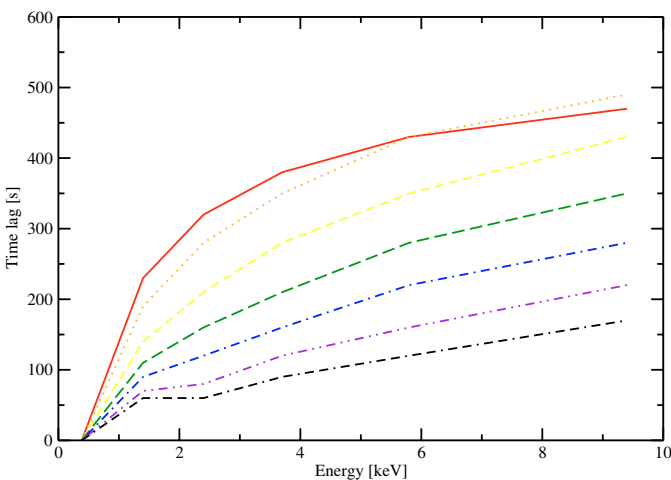

Fig. 6. Time delay curves derived for various values of the broadening $b$ in the weakly ionized/neutral reflector scenario. The normalization $\mathrm{Nb}$ of the total energy released is kept constant at $N b=1.8$. The upper curve (red, solid) represents the value $b=1.5$. From top to bottom, the value increases in steps of 0.5 for each curve and ends up at $b=4.5$ for the lowest curve (black dots/double-dashes).

\subsection{Comptonization delay versus reprocessing delay}

Ponti et al. (2004) argued that the observed delays are caused by Comptonization inside the flare source, whilst in our approach we neglect the internal delay and model the effect of the delayed reflection. To justify this method the flare has to be compact enough. The lower limit for the flare size can be estimated from the compactness parameter (see e.g. Cavaliere \& Morrison 1980; Svensson 1994; Torricelli-Ciamponi et al. 2005): $l<12$. During the 95 ks XMM-Newton observation, MCG-6-30-15 was

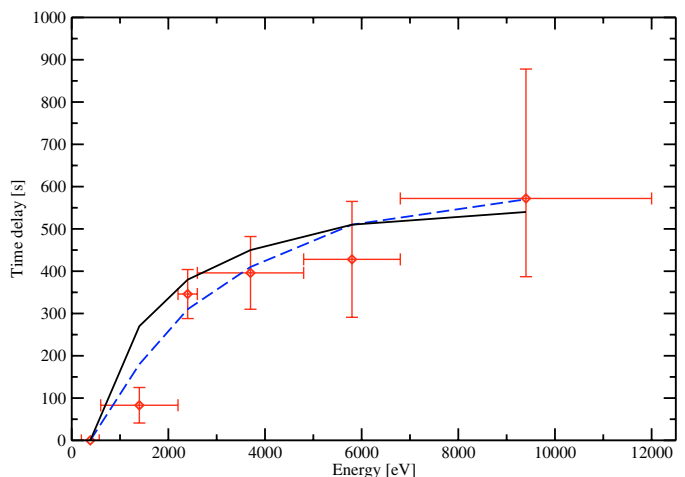

Fig. 7. Satisfying representation of the measured time delays (red diamonds with error bars) for the flare/clumps scenario. The two curves rely on the following parameterization: $\delta=1000 \mathrm{~s}, b=2.5$, and $N=1.1$ (solid, black) as well as $\delta=1000 \mathrm{~s}, b=2.5$, and $N=0.7$ (dashed, blue).

exceptionally faint, and its $2-10 \mathrm{keV}$ flux translates into a luminosity of $L=2.6 \times 10^{42} \mathrm{erg} \mathrm{s}^{-1}$. The peak luminosity of the flare is comparable to this value. Therefore, the size $D_{\mathrm{fl}}$ of the flare source has to satisfy

$D_{\mathrm{fl}}>6 \times 10^{12}\left(\frac{L}{2.6 \times 10^{42} \mathrm{erg} \mathrm{s}^{-1}}\right)\left(\frac{12}{l}\right) \quad[\mathrm{cm}]$,

so the light travel time across the flare itself can be as short as $\sim 200 \mathrm{~s}$ and this provides a lower limit for the internal delay. Unfortunately, we cannot estimate the number of scatterings 

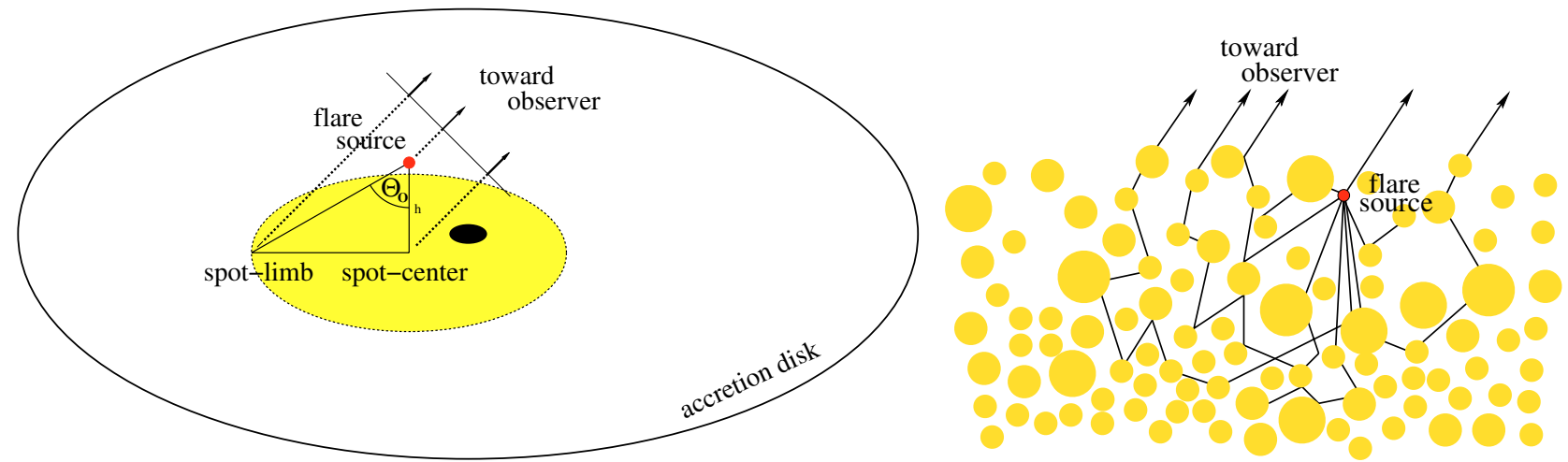

Fig. 8. Geometry of a flare. Left: a compact hard X-ray source above a reprocessing accretion disk. Right: a compact source illuminating a clumpy accretion flow. The flare is partly embedded in the flow without being completely hidden.

within the source because the observational limits for the extension of the X-ray emission in MCG-6-30-15 are not conclusive (Beppo-SAX data indicate a cut-off energy at $100-470 \mathrm{keV}$, depending on the model; Fabian et al. 2002a).

In our successful weakly ionized/neutral reflector scenario the overall shape of the delay curves is rather robust against changes of the parameters. We find that in most cases the curve slightly flattens towards larger energy separations. This flattening is a specific prediction of the model and it is encouraging that it is indicated by the data. Comptonization models on the other hand predict a linear relation in the $0.2-12 \mathrm{keV}$ band, i.e. much below the characteristic energy of the Comptonizing electrons.

\subsection{A flare located above the accretion disk}

The irradiation of a standard accretion disk by strong flare emission was studied in numerous papers (see e.g. Nayakshin et al. 2000; Nayakshin 2000; Ballantyne et al. 2001; Różańska et al. 2002; Collin et al. 2003). If the flare is located near the inner part of the disk the disk surface is strongly ionized. Relativistic modifications of the flare spectrum and Doppler shifts then explain the presence of a broadened iron $\mathrm{K} \alpha$ line (see e.g. Dovčiak et al. 2004a,b). For MCG-6-30-15 the relativistically distorted line varies less than the continuum, which can be accounted for by the light-bending model (Miniutti \& Fabian 2004). This model is also considered for explanation of the soft-excess (Crummy et al. 2006; Petrucci et al. 2006; Ponti et al. 2006) although being confronted to other approaches (Gierliński \& Done 2004, Chevallier et al. 2006).

We illustrate the simplified geometry of a flare scenario in Fig. 8 (left). The radiation is assumed to be beamed or bent toward the accretion disk and released within a cone of a certain half-opening angle $\theta_{0}$. A larger $\theta_{0}$ leads to a wider range of light travel times between the flare source and the disk, and hence the broadening of $I_{\mathrm{r}}$ increases. By varying $b$ in our model we thus change the physical picture assuming a different angular release of the primary radiation.

The geometry of the light-bending model assumes the flare source to be close to the disk axis and at varying heights above the disk surface. This lamppost geometry is justified by the idea of shocks during the relativistic jet formation (Henri \& Pelletier 1991; Martocchia et al. 2000, 2002). It is interesting to note that during the $95 \mathrm{ks}$ observation the detected line possibly became narrower with time $(\sigma=0.39 \pm 0.20$ for the detection $3000 \mathrm{~s}$ after the flare peak, and $\sigma=0.19 \pm 0.12$ for the detection $4000 \mathrm{~s}$ after the flare peak, Ponti et al. 2004), as if coming from more distant parts of the disk.
The delay model does not constrain the radial position of the flare but it infers that the flare source is elevated rather high above the disk. Assuming that the flare is triggered by magnetic reconnection, the height of the flare source above the disk surface should be equal to the disk's pressure scale height. This is implied by the equipartition between thermal and magnetic pressure as the flux tubes rise into the corona by buoyancy. An analytical fit of the disk's pressure scale height was given by Eq. (9) in Czerny \& Goosmann (2004). With $M=3.3 \times 10^{6} M_{\odot}$ and assuming a high-state accretion rate of 0.3 times the Eddington rate (Uttley et al. 2002), we obtain pressure scale heights lower than $4.5 R_{\mathrm{g}}$ for radii $R$ with $6 R_{\mathrm{g}}<R<600 R_{\mathrm{g}}$. From our delay model we infer an intrinsic time delay $\delta$, which corresponds to an elevation of the flare source of $60 R_{\mathrm{g}}$. Far from the axis, it is difficult to imagine a mechanism transporting the flux tubes so high up. Therefore, a lamppost geometry where the source is driven upwards by a central outflow would seem more likely. However, as we discuss below there is another difficulty with the model in which the primary source occurs at such a large height.

Ballantyne \& Fabian (2001) and Ballantyne et al. (2003) invoke two reflectors to explain the shape of the iron $\mathrm{K} \alpha$ line in MCG-6-30-15. They presume highly ionized reflection close to the black hole and a distant colder reflector at $\sim 70 R_{\mathrm{g}}$. In principle, this cold reflection may also form if the flare is elevated high above the disk surface as proposed in the lamp post model. The reflection is weakly ionized or neutral if the ionization parameter $\xi=L /\left(4 \pi d^{2} n\right)<10$. Herein $d$ is the distance of the flare from the disk surface with density $n$. With $L=2.6 \times 10^{42} \mathrm{erg} \mathrm{s}^{-1}$ and the local density in the disk atmosphere $n=10^{14} \mathrm{~cm}^{-3}$ we obtain the condition for an almost neutral reflection as $d>1.5 \times$ $10^{14} \mathrm{~cm}$, which corresponds to a light travel time $>1$ hour. This is more than the measured delay time during the observation and makes the scenario of a highly-elevated flare unlikely.

\subsection{The weakly ionized reflector}

The question arises where a weakly ionized/neutral reflector can be located if the flare is not allowed to be far away from the reprocessor. A possible answer is a model of cold, clumpy reflectors: the existence of magnetically confined, cold clouds in the accretion flow was suggested by Guilbert \& Rees (1988), and explored further by Celotti et al. (1992) and Kuncic et al. (1997). The interest in this model was recently revived by the detection of narrow redshifted absorption lines which suggest an inflow over a narrow range of radii (NGC 3516, Nandra et al. 1999; Mrk 509, Dadina et al. 2005; E 1821+643, Yaqoob \& Setlemitsos 2005; Q0056-363, Matt et al. 2005; PG 12111+143, 
Reeves et al. 2005; Mrk 335, Longinotti et al. 2006). The magnetically confined clumps were also recently discussed in the context of the very high state in galactic sources (see e.g. Yuan et al. 2006, and references therein). In general, partial covering remains an interesting alternative to the flat disk reflection for a number of Narrow Line Seyfert 1 galaxies (Mrk 335, Longinotti et al. 2006; 1H 0707-495, Fabian et al. 2002b).

An illustration of a clumpy reflector model is shown in Fig. 8 (right). We include some light trajectories between the flare source, the various reprocessing clouds, and the distant observer. The broadening effect on the reflection signal depends on the exact geometrical arrangement. In general, a range of light travel times around an average value is expected. Very low values of our model parameter $b$ correspond to a geometrical arrangement, where the reprocessing clouds are distributed symmetrically with respect to the flare source and to the observer. By increasing $b$ our setup drifts away from such a symmetry.

During the flare the iron $\mathrm{K} \alpha$ line complex was not detected. A strong enhancement of the line emission was seen only $\sim 3000$ s after the flare (Ponti et al. 2004). On the other hand, considerable spectral variability was seen during the flare. It is therefore possible that while the flare went off the line formed in the clumpy medium and was strongly broadened, as suggested by the parameter $b$ we obtain from the delay modeling. Then, the line could not be identified in the data. The line signature became apparent only later when the flare radiation was subsequently reprocessed by more distant and larger parts of the disk.

Our delay model requires a significantly enhanced reflection which indicates a large covering factor of the flare source with reprocessing clouds. This is consistent with the (generally) large value of the equivalent width of the iron line in MCG-6-30-15, particularly during the low flux periods (Fabian et al. 2002a). Malzac et al. (2006) pointed out that strong reprocessing can be realized by reflection in a clumpy medium, which presents an alternative to the classical light-bending models, where the flare source is elevated above the disk.

\subsection{Conclusion for a global picture}

The observed flare is an exceptional event. Our model does not require the magnetically confined, cold clouds to exist everywhere in the accretion flow. We rather suggest that MCG-6-3015 exhibits both, ionized reflection from elements of an accretion disk and colder reflection from confined clouds. The particular flare event we discuss here possibly occurred in a relatively distant region dominated by magnetic confinement and weak ionization, which correctly reproduces the observed time delays. Eventually, the magnetic confinement is correlated with the existence of the reconnecting flux tubes producing the flare.

Evidences of occasional single flares occurring at distances of the order of $\sim 10 R_{\mathrm{g}}-100 R_{\mathrm{g}}$ from the black hole are given by the signature of their orbital motion (e.g. Iwasawa et al. 2004; Turner et al. 2006). Closer in, the continuous disk may recover again due to condensation, as suggested by Liu et al. (2006) and Meyer et al. (2007). Therefore, our model is not in contradiction with general models of various phenomena in this source based on the processes taking place in the innermost region.

The qualitative results we obtain suggest to continue this line of research: to perform more sophisticated modeling of time delays and to look for suitable single flares in X-ray lightcurves of this object and of other AGN. It would be particularly important to see whether the departure of the time delay - energy bin relation from a straight line, well reproduced in our model, is a characteristic property of the phenomenon.
Acknowledgements. We thank Martine Mouchet and Michal Dovčiak for their comments on an early draft of this paper and we are grateful to Agata Różańska and Anne-Marie Dumont for their help with computing the vertical disk profile and conducting the radiative transfer. G.P. thanks Patricia Arévalo and Matteo Genghini for their help. Finally, we are grateful to the anonymous referee for detailed and constructive comments.

This work was supported by the grants 1P03D00829, and PBZ-KBN054/P03/2001 of the Polish State Committee for Scientific Research (BC), by the Laboratoire Européen Associé Astrophysique Pologne-France (BC and RWG), and by the Hans-Böckler-Stiftung (RWG). VK and RWG acknowledge support through the grants PECS 98040, IAA 300030510, GAUK 299/2004, and the Center for Theoretical Astrophysics in Prague.

\section{References}

Arévalo, P., \& Uttley, P. 2006, MNRAS, 367, 801

Arévalo, P., Papadakis, I. E., Uttley, P., McHardy, I. M., \& Brinkmann, W. 2006, MNRAS, 372, 401

Ballantyne, D. R., \& Fabian, A. C. 2001, MNRAS, 328, L11

Ballantyne, D. R., Ross, R. R., \& Fabian, A. C. 2001, MNRAS, 327, 10

Ballantyne, D. R., Vaughan, S., \& Fabian, A. C. 2003, MNRAS, 342, 239

Beckmann, V., Shrader, C. R., Gehrels, N., et al. 2005, ApJ, 634, 939

Blandford, R. D., \& Begelman, M. C. 1999, MNRAS, 303, L1

Böttcher, M., \& Liang, E. P. 1999, ApJ, 511, L37

Cavaliere, A., \& Morrison, P. 1980, ApJ, 238, L63

Celotti, A., Fabian, A. C., \& Rees, M. J. 1992, MNRAS, 255, 419

Chevallier, L., Collin, S., Dumont, A.-M., et al. 2006, A\&A, 449, 493

Collin, S., Coupé, S., Dumont, A.-M., Petrucci, P.-O., \& Różańska, A. 2003, A\&A, 400, 437

Crummy, J., Fabian, A. C., Gallo, L., \& Ross, R. R. 2006, MNRAS, 365, 1067

Czerny, B., \& Goosmann, R. 2004, A\&A, 428, 353

Dadina, M., Cappi, M., Malaguti, G., Ponti, G., \& de Rosa, A. 2005, A\&A, 442, 461

Deluit, S., \& Courvoisier, T. J.-L. 2003, A\&A, 399, 77

Dovčiak, M., Bianchi, S., Guainazzi, M., Karas, V., \& Matt, G. 2004a, MNRAS, 350,745

Dovčiak, M., Karas, V., \& Yaqoob, T. 2004b, ApJS, 153, 205

Dumont, A.-M., Abrassart, A., Collin, S. 2000, A\&A, 357, 823

Dumont, A.-M., Collin, S., Paletou, F., et al. 2003, A\&A, 407, 13

Fabian, A. C., \& Vaughan, S. 2003, MNRAS, 340, L28

Fabian, A. C., Vaughan, S., Nandra, K., et al. 2002a, MNRAS, 335, L1

Fabian, A. C., Ballantyne, D. R., Merloni, A., et al. 2002b, MNRAS, 331, L35

George, I. M., Turner, T. J., Yaqoob, T., et al. 2000, ApJ, 531, 52

Gierliński, M., \& Done, C. 2004, MNRAS, 349, L7

Goosmann, R. W., Czerny, B., Mouchet, M., et al. 2006, A\&A, 454, 741

Guilbert, P. W., \& Rees, M. J. 1988, MNRAS, 233, 475

Henri, G., \& Pelletier, G. 1991, ApJ, 383, L7

Iwasawa, K., Miniutti, G., \& Fabian, A. C. 2004, MNRAS, 355, 1073

Kaastra, J. S., et al. 2004, A\&A, 422, 97

Kazanas, D., Hua, X.-M., \& Titarchuk, L. 1997, ApJ, 480, 735

Körding, E., \& Falcke, H., 2004, A\&A, 414, 795

Kuncic, Z., Celotti, A., \& Rees, M. J. 1997, MNRAS, 284, 717

Lawrence, A., Watson, M. G., Pounds, K. A., \& Elvis, M. 1987, Nature, 325, 694

Liu, B. F., Meyer, F., \& Meyer-Hofmeister, E. 2006, A\&A, 454, L9

Longinotti, A. L., Sim, S. A., Nandra, K., \& Cappi, M. 2007, MNRAS, 374, 237

Malzac, J., Merloni, A., \& Suebsuwong, T. 2006, Astron. Nachr., 327, 985

Markowitz, A. 2005, ApJ, 635, 180

Martocchia, A., Karas, V., \& Matt, G. 2000, MNRAS, 312, 817

Martocchia, A., Matt, G., \& Karas, V. 2002, A\&A, 383, L23

Matt, G., Porquet, D., Bianchi, S., et al. 2005, A\&A, 435, 857

McHardy, I., \& Czerny, B. 1987, Nature, 325, 696

McHardy, I. M., Papadakis, I. E., Uttley, P., Page, M. J., \& Mason, K. O. 2004, MNRAS, 348, 783

McHardy, I. M., Gunn, K. F., Uttley, P., \& Goad, M. R. 2005, MNRAS, 359, 1469

Meyer, F., Liu, B. F., \& Meyer-Hofmeister, E. 2007, A\&A, 463, 1

Miniutti, G., \& Fabian, A. C. 2004, MNRAS, 349, 1435

Miniutti, G., Fabian, A. C., Goyder, R., \& Lasenby, A. N. 2003, MNRAS, 344, L22

Miyamoto, S., Kitamoto, S., Mitsuda, K., \& Dotani, T. 1988, Nature, 336, 450

Mushotzky, R. F., Done, C., \& Pounds, K. A. 1993, ARA\&A, 31, 717

Nandra, K., George, I. M., Mushotzky, R. F., Turner, T. J., \& Yaqoob, T. 1997, ApJ, 477, 602

Nandra, K., George, I. M., Mushotzky, R. F., Turner, T. J., \& Yaqoob, T. 1999, ApJ, 523, L17

Nayakshin, S. 2000, ApJ, 540, L37 
Nayakshin, S., Kazanas, D., \& Kallman, T. R. 2000, ApJ, 537, 833 Papadakis, I. E., Nandra, K., \& Kazanas, D. 2001, ApJ, 554, L133 Petrucci, P. O., et al. 2006, Astron. Nachr., 327, 1043

Piconcelli, E., Jimenez-Bailón, E., Guainazzi, M., et al. 2005, A\&A, 432, 15

Ponti, G., Cappi, M., Dadina, M., \& Malaguti, G. 2004, A\&A, 417, 451

Ponti, G., Miniutti, G., Cappi, M. et al. 2006, MNRAS, 368, 903

Poutanen, J., \& Fabian, A. C. 1999, MNRAS, 306, L31

Reeves, J. N., Pounds, K., Uttley, P., et al. 2005, ApJ, 633, L81

Reynolds, C. S., Wilms, J., Begelma, M. C., Taubert, R., \& Kendziorra, E. 2004, MNRAS, 349, 1153

Risaliti, G. 2002, A\&A, 386, 379

Różańska, A., Dumont, A.-M., Czerny, B., \& Collin, S. 2002, MNRAS, 332, 799

Shapiro, S. L., Lightman, A. P., \& Eardley, D. M. 1976, ApJ, 204, 187

Soldi, S., Beckmann, V., Bassani, L., et al. 2005, A\&A, 444, 431

Shih, D. C., Iwasawa, K., \& Fabian, A. C. 2002, MNRAS, 333, 687

Strüder, L., Briel, U., Dennerl, K., et al. 2001, A\&A, 365, L18

Sunyaev, R. A., \& Titarchuk, L. G. 1980, A\&A, 86, 121
Svensson, R. 1994, ApJS, 92, 585

Taylor, R. D., Uttley, P., \& McHardy, I. M. 2003, MNRAS, 342, L31

Torricelli-Ciamponi, G., Pietrini, P., \& Orr, A. 2005, A\&A, 438, 55

Turner, T. J., Miller, L., George, I. M., \& Reeves, J. N. 2006, A\&A, 445, 59

Uttley, P., McHardy, I. M., \& Papadakis, I. E. 2002, MNRAS, 332, 231

Vaughan, S., \& Edelson, R. 2001, ApJ, 548, 694

Vaughan, S., Edelson, R., Warwick, R. S., \& Uttley, P. 2003a, MNRAS, 345, 1271

Vaughan, S., Fabian, A. C., \& Nandra, K. 2003b, MNRAS, 339, 1237

Wilms, J., Reynolds, C. S., Begelman, M. C., et al. 2001, MNRAS, 328, L27

Yaqoob, T., \& Serlemitsos, P. 2005, ApJ, 623, 112

Yuan, F., Zdziarski, A. A., Xue, Y., \& Wu, X.-B. 2006

[arXiv: astro-ph/0608552]

Zdziarski, A. A., Johnson, W. N., Done, C., Smith, D., \& McNaron-Brown, K. 1995, ApJ, 438, L63

Życki, P. T. 2004, MNRAS, 351, 1180

Życki, P. T. 2003, MNRAS, 340, 639 